\title{
Paradigmenwechsel in der Ethikausbildung
}

\section{Rouven Porz}

PD Dr. phil., dipl. Biol., Direktion Medizin, Medizinethik und ärztliche Weiterbildung Insel Gruppe AG (Bern), Präsident der European Association of Centres of Medical Ethics (EACME) und Mitglied der Redaktion Ethik der SÄZ

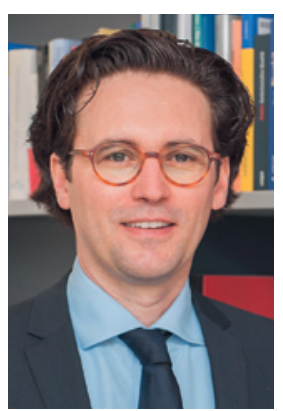

Kennen Sie Thomas S. Kuhn? Thomas S. Kuhn (19221996) war ein US-amerikanischer Wissenschaftsphilosoph, und sein Hauptwerk The Structure of Scientific Revolutions (1962) [1] scheint mir eines der wichtigsten Werke der westlichen Wissenschaftstheorie überhaupt. Mich z.B. hat das Buch dazu bewegt, nach meinem Biologiestudium nicht Biologe zu werden, sondern noch eine Geisteswissenschaft, Philosophie, zu studieren. Ein ganzes Studium wegen eines einzigen Buchs? Ja. Ein ganz neues Leben wegen eines Buchs? Ja.

Natürlich war das jetzt etwas viel Pathos, aber Sie sollen ja auch am Text dranbleiben. Und um solch ganz grosse Weichenstellungen geht es halt auch bei Kuhn. Er sagt - in einfachen Worten -, dass die Geschichte der Wissenschaften nicht eine lineare Abfolge von zunehmend mehr Wissen ist, ganz im Gegenteil: Er beschreibt die Wissenschaft als eine Folge von Phasen der «Normalwissenschaft», unterbrochen von wissenschaftlichen Revolutionen. Ein zentrales Konzept bei ihm ist das "Paradigma», und ein "Paradigmenwechsel» ist eine solche Revolution. Das Verhältnis von Forschungs- bzw. Wissenschaftsparadigmen, zwischen denen eine Revolution liegt, bezeichnet er als «inkommensurabel»: ganz und gar nicht miteinander vergleichbar. In einfachen Worten: Der Mensch, der in dem einen Paradigma dachte, dem wird es rational nicht gelingen, in dem neuen Paradigma Fuss zu fassen, inkommensurabel.

Ich glaube, so ein Paradigmenwechsel hat jetzt gerade in der Schweiz in der Ethik stattgefunden. Leise noch. Aber so zumindest in meiner Interpretation. Die SAMW hat vor kurzem ihre neuen Empfehlungen «Ethikausbildung für Gesundheitsfachpersonen» (Februar 2019) [2] herausgegeben. Es geht im Ganzen darum, der Ethikausbildung für alle Gesundheitsberufe einen professionelleren Stellenwert zu verleihen. Für mich ist das jetzt endlich ein neues Paradigma, und ich freue mich wirklich sehr darüber. Es ist allerdings sowieso schon mein Paradigma. Ich freue mich also, dass mein Weltbild zu diesen neuen Empfehlungen passt. Aber ich glaube, wir werden ein paar Menschen verlieren; Menschen, die sich diesem neuen $\mathrm{Pa}$ radigma nicht anschliessen können. Vielleicht, weil es ihnen gerade nicht kommensurabel mit ihrer eigenen Welt- bzw. Berufssicht erscheint. Ich gebe Ihnen mal zwei kurze Beispiele von Menschen, die wir jetzt in der Ethikausbildung (auf Dozentenseite) wohl verlieren werden.

Erstens: In den neuen Empfehlungen steht auf Seite 5 der harmlos klingende Satz «Ethik stellt eine eigene fachliche Disziplin dar». Das heisst, wir verlieren jetzt alle, die immer gedacht haben, Ethik könnten sie ja neben ihrer eigenen Fachkompetenz sowieso noch mitmachen, darüber schlau reden und sie unterrichten, weil sie ja selbst so gute Menschen sind. Okay dann, und vielleicht auch gut so. Ich glaube, diese Menschen haben im neuen Paradigma dann keine Lust mehr.

Zweitens Seite 7: «Die Kerninhalte der Ethikausbildung sind für alle Gesundheitsberufe gleich.» Spüren Sie die Sprengkraft? Nix mehr anfängliches grosses Auseinanderdividieren zwischen «Pflegeethik», «Medizinethik», «Physio-Ethik», «Berufsethiken» und unterschiedlichen Traditionen und Perspektiven, sondern sich einfach mal die Hand geben und sagen: «Hey, eigentlich haben wir doch im Gesundheitswesen mehr oder weniger alle die gleichen Wertüberzeugungen. Lasst uns also die Ethik mal gemeinsam denken.» Das heisst, wir verlieren alle die, die meinen, die ethische Haltung z.B. von Ärzten und Pflegenden müsste genuin unterschiedlich sein. Wir verlieren auch die, die sich in ihren Werten den anderen Berufsgruppen gegenüber überlegen fühlen. Vielleicht wollen wir genau die auch gar nicht mehr.

\footnotetext{
Literatur

1 Kuhn TS. The Structure of Scientific Revolutions. Chicago: University of Chicago Press; 1962.

2 SAMW. Ethikausbildung für Gesundheitsfachpersonen. www.samw.ch/de/Publikationen/Richtlinien.html
} 